\title{
Síntomas psicológicos y conductuales asociados a demencias: actualización terapéutica
}

\author{
Andrea Slachevsky Ch, Patricio Fuentes G.
}

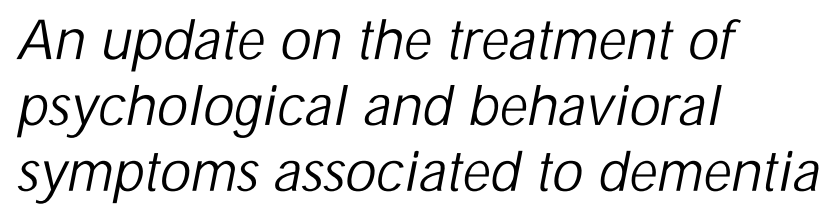

Behavioral disturbances in dementias are relevant because they are very common, they worsen patients' medical and cognitive condition, increase the caregiver burden and accelerate the admission to nursing homes. The different behavioral disturbances in dementias can appear isolated, but in most cases, patients present a combination of disturbances producing one of two main syndromes: the psychotic or the affective syndrome. Proper handling of these behavioral disturbances requires a correct characterization of the syndrome, discarding medical or environmental causes and selecting the drug with the better effectiveness and safety profile for each patient. In spite of the important number of psychotropics available, there is not enough scientific evidence about their real effectiveness and security in patients with Alzheimer's disease. This article reviews recent advances in the treatment of most common and disruptive behavioral disturbances (Rev Méd Chile 2005; 133: 1242-51).

(Key Words: Behavioral symptoms; Delirium, dementia, amnestic, cognitive disorders; Dementia)

Recibido el 10 de noviembre, 2004. Aceptado el 23 de marzo, 2005.

Unidad de Neurología Cognitiva y Demencias, Servicio de Neurología, Hospital del Salvador, Santiago, Chile.

$\mathrm{L}$ as demencias, entidades definidas como síndromes neurológicos adquiridos y caracterizados por una declinación de las capacidades cognitivas y de la autonomía, incluyen frecuentes trastornos conductuales y psicológicos, denominados síntomas psicológicos y conductuales asocia-

Correspondencia a: Patricio Fuentes G. Unidad de Neurología Cognitiva y Demencias. Servicio de Neurología, Hospital del Salvador. Av Salvador 364 Providencia, Santiago, Chile Fono: 3404231. Fax: 2066490. E mail: pfuentes@mi.cl dos a demencia (SPCD) (Tabla 1) ${ }^{1}$. Los SPCD son descritos como ssíntomas de trastornos de la percepción, del contenido del pensamiento, del ánimo y de la conducta, que frecuentemente ocurren en pacientes con demencia ${ }^{2}$. Estos síntomas pueden ser analizados individualmente 0 según dos principales combinaciones: el síndrome psicótico y el síndrome afectivo. El primero, incluye alucinaciones frecuentemente asociadas a ideas delirantes y trastornos de la conducta y el segundo se manifiesta por disforia, ansiedad, irritabilidad, agitación psicomotora, síntomas neu- 


\section{Tabla 1. Principales síntomas conductuales y psicológicos en las demencias según el Neuropsychiatric Inventory ${ }^{13}$}

\author{
Ideas delirantes \\ Alucinaciones \\ Agitación/Agresión \\ Síntoma depresivo/ Disforia \\ Ansiedad \\ Elación/Euforia \\ Apatía/Indiferencia \\ Desinhibición \\ Irritabilidad/Labilidad \\ Conductas motoras aberrantes \\ Trastornos del sueño \\ Trastornos del apetito
}

rovegetativos, tales como trastornos del sueño o del apetito, apatía y ocasionalmente ideas delirantes $^{3}$. Numerosos estudios señalan que las manifestaciones neuropsiquiátricas, más que los trastornos cognitivos, motivan la consulta inicial de los pacientes con demencia ${ }^{4}$. Las estimaciones de prevalencia de los SPCD varían ampliamente debido a la heterogeneidad de los pacientes estudiados y a las diferentes definiciones usadas ${ }^{5}$. Alrededor de dos tercios de los pacientes experimentan algún SPCD en cualquier momento de su evolución, aumentando casi a $80 \%$ en pacientes institucionalizados ${ }^{6-8}$. Estos síntomas suelen ser intermitentes y transitorios, pero una vez que un paciente los experimenta, recurren frecuentemente, con tasas de 95\% para psicosis, 93\% para agitación y 85\% para síntomas depresivos ${ }^{9}$. El SPCD predominante depende de la etapa de la enfermedad, por ejemplo, la frecuencia de síntomas depresivos disminuyen en etapas más severas, por el contrario, la agitación, agresividad y psicosis son más frecuentes en etapas tardías ${ }^{10}$. Sin embargo, en términos generales los SPCD son más frecuentes a medida que la enfermedad progresa ${ }^{4}$. Es común que los pacientes con enfermedad de Alzheimer (EA) experimenten múltiples síntomas en forma concomitante, por ejemplo, en un estudio se comunica que $29 \%$ de los pacientes experimentan dos síntomas concomitantes y 18\% tres síntomas simultáneos ${ }^{9}$. Estas manifestaciones, además, son muy importantes porque agravan los defectos cognitivos, la sobrecarga del cuidador, los costos asociados al cuidado, las comorbilidades y aceleran la institucionalización ${ }^{11}$. Los diferentes SPCD se correlacionan con anormalidades neurobiológicas, que fundamentalmente afectan a los lóbulos frontales, temporales y parietales, como se ha corroborado en estudios neuropatológicos y de neuroimágenes funcionales ${ }^{4}$.

La aparición de un importante número de nuevos agentes psicotrópicos justifica presentar una actualización del tema.

\section{GENERALIDADES SOBRE EL TRATAMIENTO}

El primer paso en la aproximación terapéutica a los SPCD, es determinar si la manifestación neuropsiquiátrica es un síntoma más dentro del conjunto de los atributos propios de la enfermedad demenciante, o más bien corresponde a la expresión clínica de un estado confusional subyacente, secundario a un proceso intercurrente, tal como una infección, constipación, deshidratación, modificación del tratamiento o a un cambio del hábitat del paciente ${ }^{12}$. Si se concluye que el síntoma neuropsiquiátrico es una manifestación más del proceso demencial propiamente tal, el primer paso es la caracterización exacta de los SPCD a través de una detallada historia clínica y del uso de instrumentos específicos, tales como el NeuroPsychiatric Inventory (NPI) ${ }^{13}$. Luego se deben identificar síntomas específicos para establecer un diagnóstico conductual sindromático, a objeto de orientar mejor las intervenciones terapéuticas. Finalmente, deben valorarse cuidadosamente las repercusiones de los SPCD sobre el paciente o el cuidador, puesto que no siempre se requerirá una intervención farmacológica. Los SPCD poco disruptivos para el medio o el paciente no requieren ser tratados ${ }^{5}$.

Las intervenciones terapéuticas de los SPCD son de dos tipos: las no farmacológicas y las farmacológicas. Actualmente, se carece de evidencia consistente acerca de la efectividad de intervenciones no farmacológicas, tales como luminoterapia, modificación ambiental o musicoterapia ${ }^{14-16}$. Sin embargo, un metaanálisis reciente de intervenciones psicosociales para cuidadores, reveló que reducen la morbilidad psicológica de aquellos y ayudan a los pacientes con demencia 
para permanecer por mayor tiempo en su propio hogar $^{17}$. Con respecto a los tratamientos con drogas, éstos deberían estar basados en evidencia científica y orientados a síndromes específicos, clínicamente significativos por su duración o severidad. En este artículo, revisaremos el tratamiento actual sólo de los SPCD más disruptivos y difíciles de manejar, tales como psicosis, agitación y trastornos del sueño y dos de los más frecuentes, el síntoma depresivo y apatía ${ }^{2,18}$.

\section{Psicosis}

La psicosis asociada a demencia incluye ideas delirantes, alucinaciones o síndromes de mala identificación, cuyas principales características están descritas en la Tabla 2. En la EA, las ideas delirantes se presentan en 30 a $40 \%$ de los pacientes y las alucinaciones en 5 a $20 \% 18,19$. Los síntomas psicóticos se suelen asociar a otros trastornos del comportamiento, como agitación motora, labilidad emocional y agresividad constituyendo el síndrome psicótico. Los principios generales del tratamiento farmacológico incluyen, primeramente, definir síntomas-objetivos específicos, tales como ideas delirantes o alucinaciones, debido a que el tratamiento para manifestaciones pobremente definidas es de beneficio limitado ${ }^{20}$. Luego deben considerarse los factores etiológicos, puesto que es diferente tratar una psicosis secundaria a una enfermedad médica o a uso inapropiado de medicamentos, a tratar una psicosis que sea el reflejo de la neuropatología de la EA. Posteriormente se pueden implementar intervenciones medio ambientales (Tabla 3); estas intervenciones son particularmente útiles para psicosis leves o conductas no agresivas ${ }^{20}$. En cuanto al tratamiento farmacológico, se deben preferir tratamientos basados en la evidencia, particularmente en ensayos realizados en pacientes con demencia, y evitar aquellos medicamentos pobremente tolerados por pacientes ancianos. Los estudios controlados con neurolépticos típicos, sugieren un efecto benéfico leve de estas clases de neurolépticos en pacientes con alteraciones conductuales en $\mathrm{EA}^{21}$. En un metaanálisis de 16 estudios con neurolépticos en pacientes con cualquier tipo de demencia y trastornos conductuales, se encontró que el promedio de pacientes que mejoraron fue de $61 \%$,

Tabla 2. Síntomas psicóticos en pacientes con Enfermedad de Alzheimer (adaptado de Sultzer, 2004)20

- $\quad$ Ideas delirantes

Persecutorios

- Alguien está robando cosas

- Alguien está viviendo en la casa

- Alguien está entrometiéndose en mis finanzas personales

- Alguien está planeando hacerme daño físico

Realizar trabajo diario diferente de la ocupación previa

Idea delirante acerca de la propia condición médica

Celotipia

Parientes muertos están vivos

Creencia de contaminación dentro de la casa

- Mala identificación

La casa no es la casa

La esposa es un impostor

Extranjeros son miembros de la familia

Las imágenes de la televisión son reales

La imagen personal en un espejo es de una persona diferente

- Alucinaciones

Visuales (ej.: ver personas en la pieza o fuera en el patio)

Escuchar la voz de miembros de familia no presentes 
Tabla 3. Intervenciones no farmacológicas para el tratamiento de las psicosis en pacientes con demencia (adaptado de International Psychogeriatrics Association) ${ }^{2}$

\begin{tabular}{|lc|}
\hline Intervención & Objetivo \\
\hline $\begin{array}{c}\text { Demostrar preocupación por el paciente y } \\
\text { permanecer cercano hasta que se calme. }\end{array}$ & $\begin{array}{c}\text { Esta intervención provee apoyo y alivia el temor } \\
\text { del paciente. }\end{array}$ \\
$\begin{array}{c}\text { Permitirle al paciente expresar sus } \\
\text { pensamientos, continuar hablándole y }\end{array}$ & Tranquilizar al paciente ayuda a disminuir \\
mantener el contacto visual. & las alucinaciones o ideas delirantes. \\
Mantener una luz nocturna en la pieza del & La agitación y la alucinación pueden \\
paciente. & empeorarse en la noche por los trastornos perceptivos. \\
Eliminar, si fuera necesario, estímulos & Estímulos externos pueden gatillar alucinaciones visuales \\
ambientales como televisión y radio. & o auditivas o impedir que el paciente perciba los \\
& estímulos importantes. \\
& \\
\hline
\end{tabular}

comparado contra $34 \%$ en el grupo placebo ${ }^{22}$. Los neurolépticos atípicos han reemplazado a los neurolépticos convencionales, debido a su más favorable perfil de efectos adversos. Sin embargo, estudios randomizados con grandes muestras de pacientes están recién comunicándose. La mayoría de los estudios señala que risperidona (1-2 mg/ día) es más efectiva que el tratamiento con placebo y que los síntomas extrapiramidales suelen presentarse a dosis más altas ${ }^{23}$. No obstante, una reciente revisión ha sugerido que los pacientes con demencia severa tratados con risperidona presentan una mayor frecuencia de eventos cerebrovasculares $^{24}$. Existe un estudio randomizado doble ciego con quetiapina (100 mg/día) de 10 semanas de duración comparado con placebo, que mostró eficacia en agitación pero no en psicosis $^{25}$.

Un estudio abierto más reciente, en 16 pacientes con EA, mostró que el uso de quetiapina (25$200 \mathrm{mg} /$ día) se tradujo en una mejoría en los síntomas psicóticos sin agravación de las manifestaciones extrapiramidales ${ }^{26}$. En cuanto a olanzapina existen dos estudios randomizados contra placebo, en pacientes con EA severa e institucionalizados, en los cuales se encontró que el tratamiento (5-10 mg/día) produjo mejoría significativa sobre el trastorno conductual global ${ }^{27,28}$. En un ensayo randomizado de aripripazol controlado con placebo, en pacientes ambulatorios con EA de severidad moderada, con alucinaciones e ideas delirantes, se encontró tendencia a mayor mejoría conductual global que con placebo; el principal efecto secundario fue sedación en un número reducido de pacientes ${ }^{29}$. No existen estudios sobre el tratamiento de las manifestaciones conductuales de la EA con ziprasidona. El uso de clozapina no está recomendado en pacientes de edad por sus posibles efectos adversos. Los anticolinesterásicos son considerados drogas psicotrópicas y como algunos trastornos neuropsiquiátricos se han asociado con disfunción colinérgica, se ha estudiado el uso de estos fármacos para el tratamiento de estos síntomas en EA. Aun cuando no se han alcanzado umbrales para significación estadística, se han demostrado efectos benéficos sobre ideas delirantes y alucinaciones con donepezilo y rivastigmina ${ }^{20}$.

\section{AgITACIÓN}

La agitación se produce en 70-90\% de los pacientes con demencia institucionalizados, la mitad de los cuales llegan a ser francamente agresivos ${ }^{30}$. El término agitación describe actividad motora, vocal o verbal inapropiada, que no tiene una explicación causal. La conducta agitada no es útil en sí 


\section{Tabla 4. Características de los principales antipsicóticos utilizados en la EA* (adaptado de Sultzer, 2004) 20}

\begin{tabular}{|c|c|c|c|c|c|c|}
\hline \multirow[t]{2}{*}{ Droga } & \multirow{2}{*}{$\begin{array}{l}\text { Dosis } \\
\text { inicialñ en EA } \\
\text { mg/día }\end{array}$} & \multirow{2}{*}{$\begin{array}{c}\text { Dosis } \\
\text { promedio en EA } \\
\mathrm{mg} / \text { día }\end{array}$} & \multicolumn{4}{|c|}{ Efectos adversos } \\
\hline & & & Sedación & $\begin{array}{c}\text { Efecto } \\
\text { hipotensor }\end{array}$ & $\begin{array}{c}\text { Efectos } \\
\text { anticolinérgicos }\end{array}$ & $\begin{array}{c}\text { Efectos } \\
\text { extrapiramidales }\end{array}$ \\
\hline Risperidona & $\begin{array}{l}0,25- \\
0,5\end{array}$ & $0,5-2$ & + & ++ & $+/-$ & + \\
\hline Olanzapina & $2,5-5$ & 5 & H & + & + & $+/-$ \\
\hline Quetiapina & 25 & $50-200$ & ++ & + & $+/-$ & - \\
\hline Ziprasidone $^{2}$ & $20 \mathrm{mg}$ & $40-120$ & + & H & $+1-$ & $+/-$ \\
\hline Aripriprazole ${ }^{2}$ & $2-5$ & $5-15$ & + & + & $+/-$ & - \\
\hline Clozapina & 12,5 & $12,5-100$ & +H & +H & H+ & - \\
\hline Haloperidol & 0,5 & $0,5-4$ & + & & & ++t \\
\hline Perfenazine & 2 & $2-16$ & + & + & ++ & ++ \\
\hline
\end{tabular}

EA: Enfermedad de Alzheimer

Efectos adversos: -: No, $+/-=$ raramente, $+=$ leve, $++=$ leve a moderado, $+++=$ severo.

misma para el paciente y afecta adversamente los esfuerzos de ayuda. Alguna de esas conductas son en sí mismas inapropiadas, mientras que otras pueden serlo debido a su intensidad, frecuencia 0 el contexto en el cual ellas se exhiben ${ }^{31}$. Como en las otras alteraciones conductuales, deben considerarse primeramente estrategias no farmacológicas, que incluyan medidas de seguridad, adecuación del ambiente físico, comunicación verbal apropiada y establecimiento de rutinas con actividades regulares y predecibles. Los medicamentos psicotrópicos deben ser usados a la menor dosis y por el período de tiempo más corto posible, monitorizando siempre los efectos secundarios. Los antipsicóticos son los fármacos mejor estudiados para la agitación y agresión, y se ha demostrado eficacia modesta pero consistente. Un metaanálisis reveló que los antipsicóticos convencionales son ligeramente efectivos ( $18 \%$ mejor que placebo) en tratar la agitación asociada a demencia, no demostrándose superioridad de ningún neuroléptico sobre otro ${ }^{21}$. No obstante, otra revisión sistemática sugirió que haloperidol era útil en el control de la agresión, pero se asociaba a importantes efectos colaterales ${ }^{32}$. Al igual que para el tratamiento de la psicosis, los antipsicóticos atípicos se están convirtiendo en el tratamiento de elección de la agresión, por ser igualmente efectivos que los agentes convencionales y con menos efectos adversos. En relación a los anticonvulsivantes, existen pocos estudios controlados. Un estudio con valproato (850 mg/día) en 56 pacientes con demencia institucionalizados mostró mejoría significativa en agitación en el grupo tratado, con buena tolerancia ${ }^{33}$. No obstante, el uso de valproato se ha limitado debido a que hay reportes de parkinsonismo reversible e incluso deterioro cognitivo con su uso crónico ${ }^{34}$. La carbamazepina (300 mg/día) mostró eficacia significativa con buen nivel de tolerabilidad en un ensayo de corto plazo en pacientes dementes institucionalizados ${ }^{35}$. Hasta la fecha, no existen estudios con otros anticonvulsivantes, aunque estudios de casos han mostrado buena respuesta con gabapentina ${ }^{36}$. Las benzodiazepinas son ampliamente usadas en pacientes con demencia, a menudo como medicación pro re nata, sin embargo, su uso se ha asociado con confusión mental, 
riesgo de caídas y desinhibición conductual. Un estudio efectuado con alprazolam mostró efectividad equivalente a haloperidol en dosis baja en el manejo de episodios disruptivos en pacientes dementes institucionalizados ${ }^{37}$. Un ensayo con buspirona (15-60 mg/día) mostró significativa mejoría en pacientes con demencia y conductas disruptivas ${ }^{38}$. Dada la alta frecuencia de síntomas depresivos asociados a la agitación en demencia, se ha estudiado la utilización de agentes antidepresivos. Un estudio con citalopram (10-40 mg/día en la noche) evidenció que se comportaba como antipsicótico en pacientes dementes no deprimi$\operatorname{dos}^{39}$. La trazodona en dosis alta-moderada (200$250 \mathrm{mg} /$ día) ha mostrado efectividad equivalente a uso de haloperidol (1-1,5 mg/día) en el control de la agitación en pacientes con demencia, presentando incluso mejor tolerabilidad ${ }^{40}$. Como ya hemos mencionado los anticolinesterásicos tienen propiedades psicotrópicas, por lo cual se recomienda, cuando la agitación es de magnitud leve, esperar durante un período razonable una eventual respuesta al agente anticolinesterásico y en caso de fracaso instaurar un tratamiento con inhibidores de recaptación de serotonina (IRSS), tales como la sertralina, citalopram o mirtazapina o con trazodona ${ }^{41}$. No se recomienda como de primera línea, la fluoxetina o paroxetina por las mayores interacciones medicamentosas. En caso de agitación importante, se recomienda iniciar el tratamiento con neurolépticos atípicos o anticonvulsivantes.

\section{TRASTORNOS DEL SUEÑO}

Sobre $50 \%$ de los cuidadores describen trastornos del sueño en los pacientes con EA, destacando despertares nocturnos frecuentes, fragmentación del sueño y agitación nocturna ${ }^{42}$. Antes de iniciar un tratamiento sintomático específico de estas alteraciones deben considerarse algunas etiologías, por ejemplo, una infección del tracto urinario o la polifarmacia podrían conducir a una disrupción conductual nocturna ${ }^{43}$. Adicionalmente, un paciente puede tener un trastorno específico del sueño, por ejemplo un síndrome de piernas inquietas 0 apnea del sueño ${ }^{43}$. En ausencia de tal trastorno específico del sueño o de una causa secundaria bien determinada, se deben intentar inicialmente medidas no farmacológicas, como restricción del sueño diurno, actividad física liviana y luminotera$\mathrm{pia}^{43}$. En caso de respuesta insuficiente a esas medidas cabe iniciar un tratamiento con drogas con propiedades hipnóticas.

Un ensayo con 60 pacientes con demencia tratados con zolpidem a dosis altas $(20 \mathrm{mg} /$ noche), mostró mejoría significativa del sueño ${ }^{44}$. No obstante, hay que tener presente que el uso de hipnóticos, incluso de vida media corta, contribuyen al riesgo de caídas nocturnas, por lo que se prefiere iniciar el tratamiento con dosis baja de un antidepresivo, que posea propiedades hipnóticas, tal como la trazodona ${ }^{45}$. Los antipsicóticos atípicos presentan también algunas propiedades hipnóticas para el manejo de la conducta agitada nocturna de estos pacientes. Un ensayo controlado doble ciego randomizado de olanzapina (5-10 mg/día) en pacientes con EA, aumentó el puntaje de somnolencia 5 veces en comparación con el grupo placebo ${ }^{46}$. En un estudio abierto, la quetiapina se asoció con somnolencia en un tercio de los pacientes y con risperidona se ha comunicado somnolencia dosis-dependiente ${ }^{43,47}$. El uso de anticolinesterásicos no se ha asociado ni a agravación ni a mejoría de los trastornos del sueño ${ }^{43}$. En resumen, ante un paciente con un trastorno del sueño de reciente inicio se debe primero descartar un factor gatillante, posteriormente se deben implementar medidas de higiene del sueño y si éstas fracasaran, debe iniciarse un tratamiento farmacológico sugiriéndose como de primera línea la trazodona (25 mg a $150 \mathrm{mg} /$ noche $)^{48,49}$. Cabe destacar que no es recomendable el uso de antihistamínicos para tratar los trastornos del sueño, debido a que en general tienen propiedades anticolinérgicas que pueden agravar la confusión mental del paciente.

\section{SÍNTOMA DEPRESIVO}

La asociación de síntoma depresivo y demencia se presenta en cuatro formas: la depresión puede anteceder a la EA, los síntomas depresivos pueden ser una manifestación propia de la EA, la depresión y la demencia cursan como entidades independientes y los trastornos cognitivos son secundarios a la depresión y no relacionados con el síndrome demencial. 
Los síntomas depresivos en la EA suele asociarse a otros síntomas neuropsiquiátricos constituyendo el síndrome afectivo, el cual afecta aproximadamente al 15 a 20\% de los pacientes en un mes cualquiera de su evolución ${ }^{3}$. Ambas formas clínicas de depresión, mayor o menor, comparten porcentajes equivalentes en su asociación con la $\mathrm{EA}^{3}$. El diagnóstico de síntomas depresivos en la EA representa un desafío para el clínico porque los síntomas depresivos en los pacientes con EA tienen una presentación fenomenológica diferente a la depresión de pacientes jóvenes, debido a que la tristeza es sólo de minutos de duración, recurre frecuentemente y la tristeza franca a menudo es reemplazada por irritabilidad, temor, preocupaciones o ansiedad. Culpabilidad y pensamiento suicida son infrecuentes, pero son un poderoso indicador de síntoma depresivo ${ }^{3}$. Las opciones terapéuticas incluyen terapia farmacológica, terapias conductuales dirigidas al paciente, sus familias o ambos y terapia electroconvulsiva. Existen pocos estudios sistemáticos que hayan evaluado cada una de estas opciones. En cuanto al tratamiento medicamentoso es fundamental evitar el uso de fármacos con propiedades anticolinérgicas, como tricíclicos, que pueden resultar en una agravación de los trastornos cognitivos. Los ensayos farmacológicos han mostrado eficacia variable de los fármacos antidepresivos estudiados, observándose mejoría entre 50 a $75 \%$ de los pacientes tratados ${ }^{3}$. Las intervenciones no farmacológicas como terapias cognitivo-conductuales también aparecen eficaces, particularmente para síntoma depresivo leve ${ }^{50}$. El uso de electroterapia convulsiva (ETC) aun cuando resulta controvertido, es también una opción para tratar síntoma depresivo. La seguridad del método no ha sido adecuadamente evaluada, pero parece ser aceptable siempre que se administre con precaución y no más de dos veces por semana ${ }^{51}$. Incluso la ETC parece mejorar la cognición cuando se usa en síntoma depresivo asociada a demencia ${ }^{52}$. Cuando se ha tomado la decisión de iniciar terapia antidepresiva se recomienda como fármacos de primera línea a sertralina o citalopram (el escitalopram, el senantiómero del citalopram es una alternativa a este último) a dosis bajas. Estas dosis deben aumentarse gradualmente hasta obtener una respuesta terapéutica, la cual puede incluso demorar- se 8 semanas $^{53}$. El tratamiento farmacológico debe mantenerse a dosis altas por varias semanas antes de considerarlo como fracaso terapéutico. Cuando se ha juzgado ausencia de respuesta terapéutica 0 no se ha conseguido beneficio con la adición de un segundo agente, que podría ser un anticonvulsivante o un antipsicótico, lo pertinente es considerar otro agente antidepresivo como la venlafaxina o mirtazapina por sus efectos noradrenérgicos o la moclobemida, un inhibidor MAO-A. La terapia electroconvulsiva puede ser una opción en ausencia de respuesta a los fármacos ${ }^{3}$.

\section{APATÍA}

La apatía es el síntoma neuropsiquiátrico más común en individuos con EA afectando aproximadamente a $70 \%$ de los pacientes en etapa moderada y sobre $90 \%$ de los pacientes en etapa avanzada ${ }^{18}$. Este síndrome se caracteriza por una pérdida de iniciativa y motivación, disminución del interés social e indiferencia emocional. El diagnóstico de apatía requiere diferenciarse de una pérdida de capacidades y de un trastorno del ánimo primario. No obstante, es importante considerar que existen síntomas que se sobreponen entre la apatía y el síntoma depresivo, tales como la pérdida de interés en las actividades, la anergia, el enlentecimiento psicomotor, la fatiga y la disminución de la instropección ${ }^{56}$. En cuanto a tratamiento farmacológico de la apatía, evidencia reciente sugiere que los propios agentes anticolinesterásicos empleados para manejar los síntomas cognitivos tienen indicación en el tratamiento de la apatía, siendo este síntoma la manifestación neuropsiquiátrica con mejor respuesta a tratamiento con anticolinesterásicos ${ }^{55}$. Un subanálisis de un estudio controlado doble ciego de 6 meses de duración con donepezilo, en pacientes con EA moderada a severa, mostró reducción estadísticamente significativa en apatía, síntoma depresivo y ansiedad. Sin embargo, en un estudio en pacientes institucionalizados con EA, esta misma droga se asoció con reducción significativa de la agitación pero no de la apatía ${ }^{56}$. Con respecto a galantamina, en un estudio de 5 meses sobre funcionamiento neuropsiquiátrico en pacientes con EA leve a moderada, el grupo tratado no mostró cambios ${ }^{57}$. A nuestro conocimiento, no se 
han estudiado otras drogas psicotrópicas en pacientes con EA y apatía.

\section{CONCLUSIONES}

Las manifestaciones neuropsiquiátricas de la EA son frecuentes en la práctica clínica y suelen asociarse sindromáticamente. Para un adecuado manejo es fundamental una correcta caracteriza-

\section{REFERENCIAS}

1. Knopman DS, Boeve BF, Petersen RC. Essentials of the proper diagnoses of mild cognitive impairment, dementia and major subtypes of dementia. Mayo Clin Proc 2003; 78: 1290-308.

2. International Psychogeriatrics Association, Behavioral and Psychological Symptoms of Dementia (BPSD). Educational Pack. 2004.

3. Lyketsos CG, LeE HB. Diagnosis and treatment of depression in Alzheimer's disease. A practical update for the clinician. Dement Geriatr Cogn Disord 2004; 17: 55-64.

4. Chung JA, Cummings JL Neurobehavioral and neuropsychiatric symptoms in Alzheimer's disease: characteristics and treatment. Neurol Clin 2000; 18: 829-46.

5. LAWLOR B. Managing behavioural and psychological symptoms in dementia. British Journal of Psychiatry 2002; 181: 463-5.

6. Haupt M, Kurz A, JanNer M. A 2-year follow-up of behavioural and psychological symptoms in Alzheimer's disease. Dement Geriatr Cogn Disord 2000; 11: 147-52.

7. Lyketsos CG, Steinberg M, Tschanz JT. Mental and behavioral disturbances in dementia: findings from Cache Country Study on Memory and Aging. Am J Psychiatry 2000; 157: 708-14.

8. Margalo-Lana M, Swann A, O’Brien J, Fairbairn A, Reichelt K, Potkins D et al. Prevalence and pharmacological management of behavioural and psychological symptoms amongst dementia sufferers living in care environments. Int J Geriatr Psychiatry 2001; 16: 39-44. ción de la conducta-problema y una identificación de su mecanismo causal. Después de descartar una causa subyacente, se recomienda tratar preferentemente las conductas disruptivas. $\mathrm{Al}$ inicio se sugiere ensayar medidas no farmacológicas y luego agentes con el mejor perfil de eficacia y seguridad, seleccionado en función del síntoma clínico más relevante. Finalmente, es válido recordar que los trastornos conductuales pueden también responder a anticolinesterásicos.

9. Levy ML, Cummings JL, Fairbanks LA, Bravi D, Calvani M, CarTa A. Longitudinal assessment of symptoms of depression, agitation, and psychosis in 181 patients with Alzheimer's disease. Am J Psychiatry 1996; 153: 1438-43.

10. Lopez OL, Becker JT, SweEt RA, KunN W, Kaufer DI, SAXTon J ET AL Psychiatric symptoms vary with the severity of dementia in probable Alzheimer's disease. J Neuropsychiatry Clin Neurosci 2003; 15: 346-53.

11. Cummings JL Alzheimer's disease. N Engl J Med 2004; 351: 56-67.

12. Taragano F, Lyketsos C. En: Demencia: Aproximacion multidisciplinaria. Mangone C.A., Allegri R.L and Ollari J.A., Editors. 1997, Sagitario: Buenos Aires.

13. Cummings JL, Mega M, Gray K, Rosenberg-Thompson S, CARUSI DA, GornBeIn J. The Neuropsychiatric Inventory: comprehensive assessment of psychopathology in dementia. Neurology 1994; 44: 2308-14.

14. Forbes D, Morgan DG, Bangma J, Peacock S, Peuetier $\mathrm{N}$, ADAMSON J. Light therapy for managing sleep, behaviour, and mood disturbances in dementia. Cochrane Database Syst Rev 2004: CD003946.

15. Price JD, Hermans DG, Grimiey Evans J. Subjective barriers to prevent wandering of cognitively impaired people. Cochrane Database Syst Rev 2000: CD001932.

16. Vink AC, Birks JS, Bruinsma MS, Scholten RJ. Music therapy for people with dementia. Cochrane Database Syst Rev 2004: CD003477.

17. Brodaty H, Green A, Koschera A. Meta-analysis of psychosocial interventions for caregivers of people with dementia. J Am Geriatr Soc 2003; 51: 657-64. 
18. Mega MS, Cummings JL, Fiorello T, Gornbein J. The spectrum of behavioral changes in Alzheimer's disease. Neurology 1996; 46: 130-5.

19. Paulsen JS, Ready RE, Stout JC, Salmon DP, Thal LJ, GRANT I ET aL. Neurobehaviors and psychotic symptoms in Alzheimer's disease. J Int Neuropsychol Soc 2000; 6: 815-20.

20. Sultzer DL Psychosis and antipsychotic medications in Alzheimer's disease: clinical management and research perspectives. Dement Geriatr Cogn Disord 2004; 17: 78-90.

21. SCHNeider LS, PoLock VE AND Lyness SA. A metaanalysis of controlled trials of neuroleptic treatment in dementia. J Am Geriatr Soc 1990; 38: 553-63.

22. Lanctot KL, Best TS, Mittmann N, Lu BA, Oh PI, EINARSON TR ET AL. Efficacy and safety of neuroleptics in behavioral disorders associated with dementia. J Clin Psychiatry 1998; 59: 550-61; quiz 562-3.

23. De Deyn PP, Katz IR, Brodaty H, Lyons B, GreEnSPAN A, BurNs A. Management of agitation aggression and psychosis associated with dementia: A pooled analysis including three randomized, placebo-controlled doble blind trials in morning home residents treated with risperidone. Clin Neurol Neurosurg 2005; 107: 497-508.

24. Mowat D, Fowle D, MacEwan T. CSM warning on atypical psychotics and stroke may be detrimental for dementia. BMJ 2004; 328: 1262.

25. TARIOT PN, IsmaIL MS. Use of quetiapine in elderly patients. J Clin Psychiatry 2002; 63 Suppl 13: 216.

26. Fujikana $\mathrm{T}$, Takahashi $\mathrm{T}$, Kinoshita A, Kajiyama $\mathrm{H}$, KuRATA A, Yamashita H et al. Quetiapine treatment for behavioral and psychological symptoms in patients with senile dementia of Alzheimer type. Neuropsychobiology 2004; 49: 201-4.

27. De Deyn PP, Carrasco MM, Deberdt W, Jeandel C, Hay DP, Feldman PD et al. Olanzapine versus placebo in the treatment of psychosis with or without associated behavioral disturbances in patients with Alzheimer's disease. Int J Geriatr Psychiatry 2004; 19: 115-26.

28. Street JS, Clark WS, Kadam DL, Mitan SJ, Jular BE, FeLdman PD ET AL. Long-term efficacy of olanzapine in the control of psychotic and behavioral symptoms in nursing home patients with Alzheimer's dementia. Int J Geriatr Psychiatry 2001; 16 Suppl 1: S62-70.
29. De Deyn PP, Jeste DV, Swanink R, Kestic D, Breder C. Aripiprazole for the treatment of pychosis in patients with Alzheimer's disease: A randomized, placebo-controlled study. J Clin Psychopharmacol 2005; 25: 463-7..

30. Cohen-Mansfield J. Assessment of agitation. Int Psychogeriatr 1996; 8: 233-45.

31. Cohen-Mansfield J, Marx M, Rosenthal A. A description of agitation in a nursing home. J Gerontol 1989; 44: M77-84.

32. Lonergan E, LuXenberg J, Colford J. Haloperidol for agitation in dementia. Cochrane Database Syst Rev, 2002: CD002852.

33. Porsteinsson A, Tariot P, Jakimmovich L, Kowalski N, Holt C, ERB R. Valproate therapy for agitation in dementia: open-label extension of a double-blind trial. Am J Geriatr Psychiatry 2003; 11: 434-40.

34. Armon C, Shin C, Miuer P, Carwile S, Browne E, EDINGER J. Reversible parkinsoninsm and cognitive impairment with chronic valproate use. Neurology 1996; 47: 626-35.

35. Tariot PN, Erb R, Podgorski CA, Cox C, Patel S, JAKIMOVICH L ET AL. Efficacy and tolerability of carbamazepine for agitation and aggression in dementia. Am J Psychiatry 1998; 155: 54-61.

36. GRAY KF. Managing agitation and difficult behavior in dementia. Clin Geriatr Med 2004; 20: 69-82.

37. Christensen DB, BeNField WR. Alprazolam as an alternative to low-dose haloperidol in older, cognitively impaired nursing facility patients. J Am Geriatr Soc 1998; 46: 620-5.

38. COOPER J. Buspirone for anxiety and agitation in dementia. Journal of Psychiatry Neurosciences 2003; 28: 469.

39. Polock BG, Mulsant BH, Rosen J, Sweet RA, Mazumdar S, Bharucha A et al. Comparison of citalopram, perphenazine, and placebo for the acute treatment of psychosis and behavioral disturbances in hospitalized, demented patients. Am J Psychiatry 2002; 159: 460-5.

40. Sultzer DL, Gray KF, Gunay I, Berisford MA, MAHLER ME. A double-blind comparison of trazodone and haloperidol for treatment of agitation in patients with dementia. Am J Geriatr Psychiatry 1997; 5: 60-9.

41. Trinh NH, Hoblyn J, Mohanty S, Yafre K. Efficacy of cholinesterase inhibitors in the treatment of neuropsychiatric symptoms and functional impairment in Alzheimer disease: a meta-analysis. JAMA 2003; 289: 210-6. 
42. Swearer JM, Rachman D, O’Donnell BF. Troublesome and disruptive behaviors in dementia. J Am Geriatr Soc 1988: 784-90.

43. Buwise DL. Sleep disorders in Alzheimer's disease and other dementias. Clin Cornerstone 2004; 6 Suppl 1A: S16-28.

44. Shaw SH, Curson H, Coquelin JP. A double-blind, comparative study of zolpidem and phcebo in the treatment of insomnia in elderly psychiatric in-patients. J Int Med Res 1992; 20: 150-61.

45. Mendez MF, Cummings BJ. Dementia. A clinical approach. 3 ed. 2003, Philadelphia: ButterworthHeinemann.

46. Street JS, Clark WS, Gannon KS, Cummings JL, Bymaster FP, TAMURA RN et al. Olanzapine treatment of psychotic and behavioral symptoms in patients with Alzheimer disease in nursing care facilities: a double-blind, randomized, placebocontrolled trial. The HGEU Study Group. Arch Gen Psychiatry 2000; 57: 968-76.

47. McManus D, Arvanitis L, Kowalcyk B. Quetiapine, a novel antipsychotic: experience on elderly patiens with psychotics disorders. Seroquel Trial 48 Study Group. J Clin Psychiatry 1999; 60: 292-8.

48. LEBERT F. Serotonin reuptake inhibitors in depression of Alzheimer's disease and other dementias. Presse Med 2003; 32: 1181-6.

49. SADAVOY J. Psychotropic drugs and the elderly fast facts. 2004, New York: Norton.

50. Teri L, Logsdon RG, Uомoto J, McCurRy SM. Behavioral treatment of depression in dementia patients: a controlled clinical trial. J Gerontol B Psychol Sci Soc SCi 1997; 52: P159-66.
51. KaTZ IR. Diagnosis and treatment of depression in patients with Alzheimer's disease and other dementias. J Clin Psychiatry 1998; 59 Suppl 9: 38-44.

52. RAO V, LyKeTsos CG. The benefits and risks of ECT for patients with primary dementia who also suffer from depression. Int J Geriatr Psychiatry 2000; 15: 729-35.

53. Lyketsos CG, Sheppard JM, Steele CD, Kopunek $S$, Steinberg M, BaKer AS et al. Randomized, placebo-controlled, double-blind clinical trial of sertraline in the treatment of depression complicating Alzheimer's disease: initial results from the Depression in Alzheimer's Disease study. Am J Psychiatry 2000; 157: 1686-9.

54. Boyle PA, MaLoy PF. Treating Apathy in Alzheimer's disease. Dement Geriatr Cogn Disord 2004; 17: 91-9.

55. Gauthier S, Feldman H, Hecker J, Velias B, Ames D, SubBiaH P ET AL. Efficacy of donepezil on behavioral symptoms in patients with moderate to severe Alzheimer's disease. Int Psychogeriatr 2002; 14: 389-404.

56. TARiot PN, Cummings JL, Katz IR, Mintzer J, Perdomo CA, Schwam EM et al. A randomized, double-blind, placebo-controlled study of the efficacy and safety of donepezil in patients with Alzheimer's disease in the nursing home setting. J Am Geriatr Soc 2001; 49: 1590-9.

57. WiLCock GK, LLLENFELD S, Gaens E. Efficacy and safety of galantamine in patients with mild to moderate Alzheimer's disease: multicentre randornised controlled trial. Galantamine International-1 Study Group. BMJ 2000; 321: 1445-9.. 